made only after the accumulation of such a large amount of data as this. The small peaks at the right of figure 3 indicate a slight tendency for the observed counts to be influenced by effects other than statistical fluctuations. However, it must be remembered that the data include instances where either counters or scaling circuits have gone bad and have evidenced a need for repair or replacement, so that this slight excess is to be expected. It is believed that this is the first time that such a large collection of Chi square values has been obtained from the ratio of the error variance of Latin squares to the expected (Poisson) variance.

It is well known that individual measurements of radioactive disintegrations follow a Poisson law, but the close agreement between the experimental and theoretical curves illustrates one further point: that the Latin square arrangement has been completely successful in eliminating extraneous, nonPoisson fluctuations from the measurements.

The author thanks W. J. Youden and J. M. Cameron, of the Statistical Engineering Section, for their suggestions as to the use of the Latin squares and for many valuable hours of discussion; and Margaret Selgin and Lucy Cavallo, of the Radioactivity Section, who performed the calculations summarized in figure 3 .

Washington, July 26, 1950.

\title{
A Problem in Precision Cam Design
}

\section{By Joseph Blum}

\begin{abstract}
This paper proposes an analytic method for determining the profile of a cam required in a device that demands high precision. The method is applicable whenever the equation for the profile can be obtained by making a less stringent assumption, namely, that the follower makes contact at a fixed point of its extremity. The true profile can then be determined numerically by considering it as the envelope of a family of curves.
\end{abstract}

The design of an X-ray spectrograph often requires that a constant rate of oscillation be maintained in the crystal holder. The crystal rotates through an angle $\beta$ (the angle of travel), reverses its direction and then proceeds with the same angular velocity. This type of motion may be obtained from a cam rotating with constant angular velocity, $\omega$. An analytic method for computing the profile of the cam is presented in this paper which extends, in a certain sense, the application of a paper written by J. B. Friauf; ${ }^{1}$ the notation therein will be preserved to a considerable extent.

\footnotetext{
1 J. B. Friauf, The design of a cam for an X-ray spectrograph, J. Opt. Soc. Am Rev. Sci. Instr. 11, 289 to 296 (1925).
}

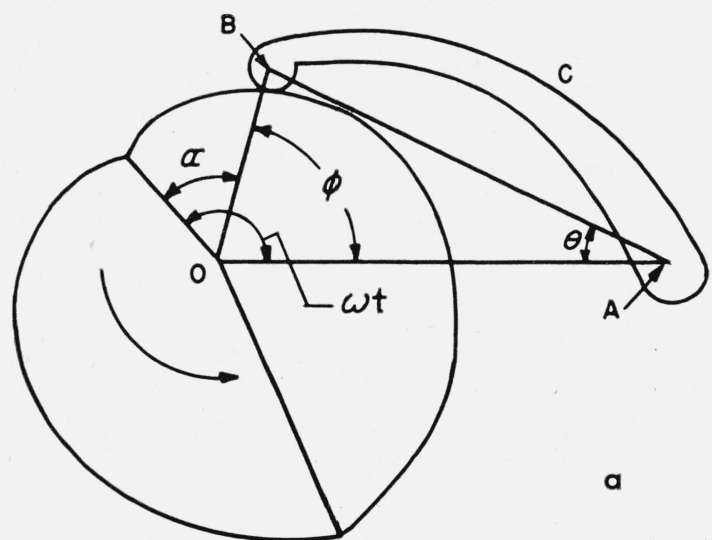

In figure 1, a, $O$ represents the axis about which the cam rotates, $A$ the axis about which the crystal holder rotates, and $B$ the center of the ball at the end of the follower. The follower $A C B$ makes contact with the cam and serves to rotate the crystal holder. The distances $A O$ and $A B$ are equal; denote this common length by $R$. Let $r_{1}$ and $r_{2}$ be, respectively, the least and greatest radii measured from $O$ to the point $B$; let $r$ be the variable radius, $O B$ corresponding to the angle $\alpha$, which $r$ makes with the least radius $r_{1}$.

Denote the time by $t$ and let $t=0$ represent the time when the least radius coincides with the line segment extending from $O$ to $A$. Then the angle

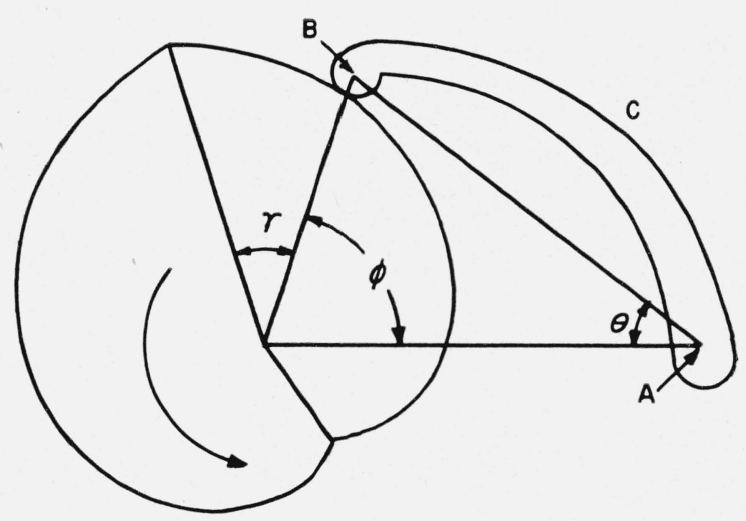

b

Figure 1. 
between $r_{1}$ and $O A$ is given by $\omega t$. Let $\theta$ denote angle $O A B$ and $\phi$ the angle $A O B$. Unless otherwise indicated, all angles are in radians.

From figure 1, a, it is clear that

$$
\sin \frac{\theta}{2}=\frac{r}{2 R}
$$

from which it follows that the smallest angle $\theta_{1}$ occurs when $r=r_{1}$ and the largest angle $\theta_{2}$ when $r=r_{2}$, or

$$
\sin \frac{\theta_{1}}{2}=\frac{r_{1}}{2 R}
$$

and

$$
\sin \frac{\theta_{2}}{2}=\frac{r_{2}}{2 R}
$$

It is also clear that $\beta=\theta_{2}-\theta_{1}$. Differentiating eq 1 yields

$$
\begin{aligned}
& \cos \frac{\theta}{2} \frac{d \theta}{d t}=\frac{1}{R} \frac{d r}{d t}=\frac{1}{R} \frac{d r}{d \alpha} \frac{d \alpha}{d t} . \\
& \text { Since } \alpha=\omega t-\phi, \frac{d \alpha}{d t}=\omega-\frac{d \phi}{d t} .
\end{aligned}
$$

Substituting this into eq 4 yields

$$
\cos \frac{\theta}{2} \frac{d \theta}{d t}=\frac{1}{R} \frac{d r}{d \alpha}\left(\omega-\frac{d \phi}{d t}\right) .
$$

Again, since $\phi=\frac{1}{2}(\pi-\theta),(d \phi / d t=-1 / 2(d \theta / d t)$, and substituting this into eq 5 results in

$$
\cos \frac{\theta}{2} \frac{d \theta}{d t}=\frac{1}{R} \frac{d r}{d \alpha}\left(\omega+\frac{1}{2} \frac{d \theta}{d t}\right)
$$

The follower rotates through the angle $\beta$ during the same time interval that it takes the cam to rotate through $\pi$ radians. Therefore, as the respective angular velocities are constant,

$$
\frac{\frac{d \theta}{d t}}{\omega}=\frac{\beta}{\pi}, \text { or } \frac{d \theta}{d t}=\frac{\beta \omega}{\pi} .
$$

Substituting this into eq 6 gives

$$
\cos \frac{\theta}{2}=\frac{1}{R} \frac{d r}{d \alpha}\left(\frac{\pi}{\beta}+\frac{1}{2}\right)
$$

From eq $1 \cos (\theta / 2)=(1 / 2 R) \sqrt{4 R^{2}-r^{2}}$. Substituting this into eq 7 and simplifying yields

$$
\frac{2 d r}{\sqrt{4 R^{2}-r^{2}}}=\frac{d \alpha}{\beta_{1}}
$$

where $\beta_{1}=\pi / \beta+1 / 2$.

After integrating this equation there is obtained

$$
2 \sin ^{-1} \frac{r}{2 R}=\frac{\alpha}{\beta_{1}}+C_{1}
$$

where $C_{1}$ is the constant of integration.
When $\alpha=0, r=r_{1}$; solving for $C_{1}$ results in $C_{1}=2$ $\sin ^{-1} r_{1} / 2 R=\theta_{1}$. Equation 9 can be expressed in the form

$$
r=2 R \sin \frac{1}{2}\left\{\frac{\alpha}{\beta_{1}}+\theta_{1}\right\} .
$$

The greatest radius, $r_{2}$, occurs when $\alpha=\pi+\beta / 2$, which may be vertified by substituting into eq 10 .

If the ball that makes contact with the cam were sufficiently small, then eq 10 would give one part of the cam's contour, and a similar equation would give the remaining part. Frequently, however, this condition is not met, and the method given in the reference in footnote 1 must be extended to secure a high level of precision. Consider then the family of circles of radius $\rho$, whose centers lie on the curve given by eq 10 . The envelope of this family consists of two distinct curves; the one that lies nearer the center of curvature of eq 10 coincides with the contour of the cam. This envelope can be obtained by a numerical determination of the $C$-discriminant. ${ }^{2}$

Let the curve of eq 10 be given in Cartesian coordinates by

$$
y=f(x) .
$$

Let $(x, y)$ be an arbitrary point on this curve. The equation of the circle with radius $\rho$ and center $(x, y)$ is given by

$$
(X-x)^{2}+(Y-y)^{2}=\rho^{2} .
$$

Substituting $y=f(x)$ into eq 12 , there is obtained a one-parameter family of circles

$$
\{X-x\}^{2}+\{Y-f(x)\}^{2}=\rho^{2} .
$$

Differentiating eq 13 with respect to $x$ yields

$$
\{X-x\}+f^{\prime}(x)\{Y-f(x)\}=0 .
$$

The $C$-discriminant is obtained by solving eq 13 and 14 simultaneously for a discrete set of values of the parameter $x$. The method outlined below yields the numerical solution:

1. For a selected set of values $\alpha_{i}$; determine the values of $r_{i}$ by using eq 10 . Denote these points by $P_{i}$.

2. Let $\tau_{i}$ be the angle of inclination of the tangent line at $P_{i}$; let $\psi_{i}$ be the angle between the radius $r_{i}$ and the tangent line. Then, since $\tau_{i}=\alpha_{i}+\psi_{i}$,

$$
f^{\prime}(x)=\tan \tau_{i}=\frac{\tan \alpha_{i}+\tan \psi_{i}}{1-\tan \alpha_{i} \tan \psi_{i}} .
$$

Now $\tan \psi_{i}=r_{i} / r_{i}^{\prime}$, where $r_{i}^{\prime}$ is the derivative $d r / d \alpha$ evaluated at $P_{i}$. This leads to

$$
\tan \psi_{i}=2 \beta_{1} \tan \frac{1}{2}\left(\frac{\alpha_{i}}{\beta_{i}}+\theta_{1}\right) .
$$

\footnotetext{
2 E. L. Ince, Ordinary differential equations, p. 85 to 86 (Longmans, Green \& Co., New York, N. Y., 1944).
} 
These relations enable one to compute the value of $f^{\prime}(x)$.

3. The solution of eq 13 and 14 is easily obtained in the form

$$
\left.\begin{array}{l}
X_{i}=x_{i} \pm \frac{\rho f^{\prime}\left(x_{i}\right)}{\sqrt{1+\left\{f^{\prime}\left(x_{i}\right)\right\}^{2}}} \\
Y_{i}=y_{i}+\frac{\rho}{\sqrt{1+\left\{f^{\prime}\left(x_{i}\right)\right\}^{2}}}
\end{array}\right\}
$$

The points $\left(X_{i}, Y_{i}\right)$ are on the envelope, as required.

To obtain the other part of the cam, consider that phase of the motion in which the angle $\theta$ decreases (fig. 1, b). For this phase,

$$
\frac{d \theta}{d t}=-\frac{\beta \omega}{\pi} .
$$

Substituting this into eq 6 results in

$$
\cos \frac{\theta}{2}=\frac{1}{R} \frac{d r}{d \gamma}\left(\frac{1}{2}-\frac{\pi}{\beta}\right)
$$

where $\gamma$ denotes the angle that $r$ makes with the greatest radius $r_{2}$ (see fig $\left.1, \mathrm{~b}\right)$. Substituting $\cos (\theta / 2)=$ $(1 / 2 R) \sqrt{4 R^{2}-r^{2}}$ into eq (16) and simplifying gives

$$
\frac{2 d r}{\sqrt{4 R^{2}-r^{2}}}=\frac{d \gamma}{\beta_{2}}
$$

where $\beta_{2}=1 / 2--\pi / \beta$.

When eq 17 is integrated there results

$$
2 \sin ^{-1} \frac{r}{2 R}=\frac{\gamma}{\beta_{2}}+C_{2},
$$

where $C_{2}$ is the constant of integration. When $\gamma=0$, $r=r_{2}$; solving for $C_{2}$ yields $C_{2}=2 \sin ^{-1} r_{2} / 2 R=\theta_{2}$. Equation 18 can be expressed in the form

$$
r=2 R \sin \frac{1}{2}\left(\frac{\gamma}{\beta_{2}}+\theta_{2}\right) .
$$

As a check, when $\gamma=\pi-\beta / 2, r=2 R$ sin $\frac{1}{2}\left(\theta_{2}-\beta\right)=$ $2 R \sin 1 / 2 \quad \theta_{1}=r_{1}$. The envelope of the family of

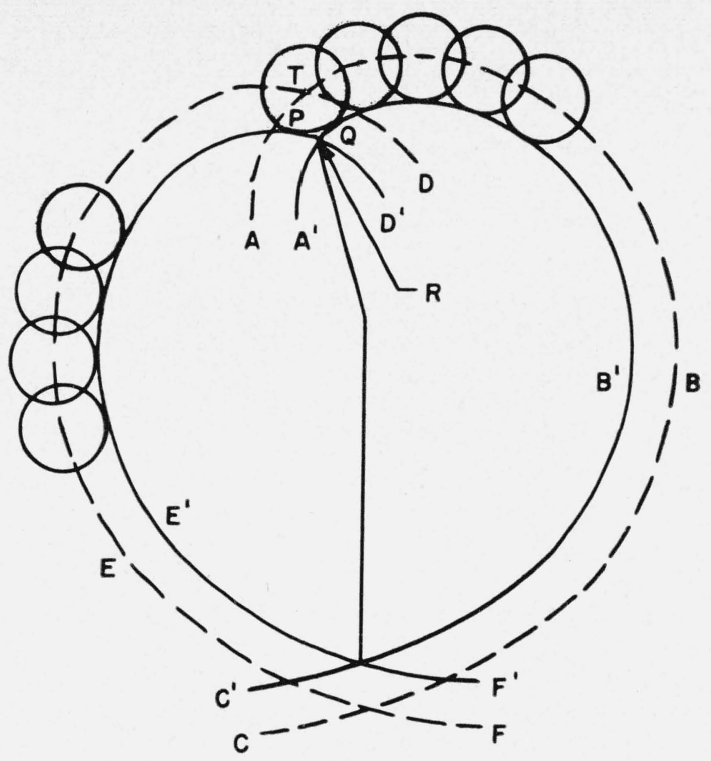

Figure 2.

circles with centers on the curve (19) and radius $\rho$ leads to the remainder of the contour of the cam.

To complete the analysis, consider figure 2 . The $\operatorname{arc} A B C$ is the locus of eq 10 , and arc $A^{\prime} R B^{\prime} C^{\prime}$ is its envelope. Similarly, the arc $D E F$ is the locus of eq 19 ; $\operatorname{arc} D^{\prime} R E^{\prime} F^{\prime}$ is its envelope and may be found numerically by following the procedure outlined above for eq 10. The high point of the cam is located numerically by solving for the intersection of the two envelopes. The low portion of the cam does not coincide with the envelope, as is evident from the figure. Let $T$ be the point of intersection of the curves 10 ard 19. The circular are, $P$ ? , with center at $T$ and radius $\rho$ constitutes the low portion of the cam. These considerations enable one to complete the numerical solution of the cam design.

In summary, it should be noted that the essential idea in this approach is to first find the equation of the contour, assuming that the follower makes contact at a fixed point of its extremity; then, by using the true form of the follower, obtain the actual cam contour by determining the envelope of a family of curves. It is evident that the analytic method applied in this particular case may be used in other problems of precise cam design.

Washington, August 4, 1950. 